\title{
Set-Valued Stochastic Equation with Set-Valued Square Integrable Martingale
}

\author{
Jun-Gang Li ${ }^{1}$, Shi-Qing Zheng ${ }^{2}$ \\ ${ }^{1}$ Department of Statistics North China University of Technology, Beijing 100144, China. \\ ${ }^{2}$ Department of Statistics North China University of Technology, Beijing 100144, China. \\ 1jgli@ncut.edu.cn, ${ }^{2} 1416922582 @ q q . c o m$
}

\begin{abstract}
In this paper, we shall introduce the stochastic integral of a stochastic process with respect to set-valued square integrable martingale. Then we shall give the Aumann integral measurable theorem, and give the set-valued stochastic Lebesgue integral and set-valued square integrable martingale integral equation. The existence and uniqueness of solution to set-valued stochastic integral equation are proved. The discussion will be useful in optimal control and mathematical finance in psychological factors.
\end{abstract}

\section{Introduction}

Set-valued theory is used in optimal control(cf.[1]), mathematical finance(cf. [2]), fixed point theory(cf. [3]). Set-valued and fuzzy set-valued theory can be used to account for psychological factors(cf.[4,5]).In [6], stochastic control problems are discussed by stochastic integral with respect to set-valued square integral martingales. M. Malinowski et al. discussed the setvalued stochastic integral driven by semi-martingale and the set-valued stochastic differential equations driven by semi-martingale in [7]. J. Li et al. discussed setvalued stochastic Lebesgue integral and set-valued stochastic differential equaton in $[8,9,10]$. Puri and Ralescu defined fuzzy set-valued martingales and proved convergence theorems of fuzzy set-valued martingales in [11]. J. Li et al. discussed the space of fuzzy set-valued square integrable martingales in [12] and fuzzy set-valued stochastic Lebesgue integral in [13].

In this paper, we shall give the set-valued stochastic integral equation :

$$
F(t)=F(0)+(L) \int_{0}^{t} b(s, F(s)) d s+(M) \int_{0}^{t} \sigma(s, F(s)) d G(s)
$$

the first integral is set-valued stochastic Lebesgue integral (see $[9,10]$ ), the second integral is set-valued square integrable martingale integral (see [6]). Aumann integral measurable theorem of the second integral shall be given. The existence and uniqueness of the solution to the equation shall be proved.

We organize our paper as follows: in Section 2, we shall introduce some necessary notations, definitions, and results about set-valued stochastic variables and martingales.
Furthermore, we shall give the Aumann integral measurable theorem. In Section 3, we shall give the set-valued stochastic integral equation with respect to set-valued square integrable martingale, and prove the existence and uniqueness of the solution to the set-valued stochastic integral equation.

\section{Preliminary on Set-Valued Random Variables and Martingales}

Throughout this paper, assume that $(\Omega, \mathcal{A}, \mu)$ is a complete probability space, the $\sigma$-field filtration $\left\{\mathcal{A}_{t}\right.$ : $t \in I\}$ satisfies the usual conditions (i.e. Containing all nullsets, non-decreasing and right continuous), $I=[0, T]$ with $T>0, R$ is the set of all real numbers, $N$ is the set of all natural numbers, $R^{d}$ is the $d$-dimensional Euclidean space with usual norm $\|\times\|$, $\mathfrak{B}(E)$ is the Borel field of the space $E$. Let $K\left(R^{d}\right)$ be the family of all nonempty, closed subsets of $R^{d}$. Let $K_{c}\left(R^{d}\right)$ (resp. $\left.K_{k}\left(R^{d}\right), K_{k c}\left(R^{d}\right)\right)$ be the family of all nonempty closed convex (resp. compact, compact convex) subsets of $R^{d}$. For any $x \hat{\mathrm{I}} R^{d}, A, B$ are nonempty subsets of $R^{d}$, define the distance between $x$ and $A$,

$$
\begin{gathered}
d(x, A)=\inf _{y \in A}\|x-y\|, \\
A+B=\{a+b: a \in A, b \in B\}, \\
A \ominus B=\left\{x \in R^{d}: x+B \subset A\right\} .
\end{gathered}
$$

The Hausdorff metric on $K\left(R^{d}\right)$ is defined as 


$$
d_{H}(A, B)=\max \left\{\sup _{a \text { 过 } 4} d(a, B), \sup _{b B} d(b, A)\right\}
$$

for $A, B \in K\left(R^{d}\right)$. For $B \in K\left(R^{d}\right)$, define

$\|B\|_{K}=d_{H}(0, B)=\sup _{a \mathrm{l} B}\|a\|$.

If $F:(\mathrm{W}, \mathcal{A}) \circledR K\left(R^{d}\right)$ satisfies that for any open set

$O \subset R^{d}, F^{-1}(O)=\{\omega \in \Omega: F(\omega) \bigcap O \neq \Phi\} \in \mathcal{A}$ then $F$ is called $\mathcal{A}$-measurable (or a set-valued random variable, random set, multivalued function.).

Let $f=\left\{f(t), \mathcal{A}_{t}, t \in I\right\}$ be a $R^{d}$-valued adapted stochastic process. Let $L^{p}\left[\mathrm{~W}, \mathcal{A}_{t}, m, R^{d}\right]$ be the set of $R^{d}$-valued $\mathcal{A}_{t}$-measurable random variable $x$ with $E\left[\|x\|^{p}\right]<イ(1 \quad p<?)$, write $\|x\|_{p}=\left[E\left[\|x\|^{p}\right]\right]^{1 / p}$.

Let

$\mathcal{A})=\left\{f \in L^{p}\left[\Omega, \mathcal{A}, \mu: R^{d}\right]: f(\omega) \in F(\omega)\right.$, a.e. $\left.\omega \in \Omega\right\}$

For short, denoted by $S_{F}^{p}$. A set-valued random variable $F: \Omega \rightarrow K\left(R^{d}\right)$ is called integrable if $S_{F}^{1}$ is nonempty. $F$ is $L^{p}$-bounded if and only if the real-valued random variable $\|F\|_{K} \in L^{p}[\Omega ; R]$ If $F$ is $L^{1}$ bounded, then $F$ is also called integrably bounded. Let $L^{p}\left[\mathrm{~W}, \mathcal{F}, m, K\left(R^{d}\right)\right]$ be the family of all $K\left(R^{d}\right)$ valued $L^{p}$-bounded $\mathcal{F}$-measurable random variables. Similarly, we have notations $L^{p}\left[\mathrm{~W}, \mathcal{F}, m, K_{c}\left(R^{d}\right)\right]$, $L^{p}\left[\mathrm{~W}, \mathcal{F}, m, K_{k c}\left(R^{d}\right)\right], L^{p}\left[\mathrm{~W}, K\left(R^{d}\right)\right]$ and so on. A set-valued stochastic process $F=\left\{F(t), \mathcal{A}_{t}, t \hat{\mathrm{I}} I\right\} \quad$ is called a set-valued martingale if

(i) $F=\left\{F(t), \mathcal{A}_{t}, t \hat{\mathrm{I}} I\right\}$ is adapted and for any $t \hat{\mathrm{I}} I, F(t)$ is $L^{1}$-bounded;

(ii) for any $t^{3} s, t \hat{\mathrm{I}} I, E\left[F(t) \mid \mathcal{A}_{s}\right]=F(s)$, a.e. $(\mu)$.

A set-valued martingale $F=\left\{F(t), \mathcal{A}_{t}, t \hat{\mathrm{I}} I\right\}$ is call-ed square integrable, if $\sup _{t \hat{1} I} E\left[\|F(t)\|_{K}^{2}\right]<$ ? .

Denote $C M S(F)$ as the set of $R^{d}$-valued continuous martingale selections of set-valued square integrable martingale $F$.

Concerning more notations, definitions and more results of set-valued random variable, set-valued martingales, readers could refer to $[6,14]$.

Definition (cf.[6]) Assume $F=\left\{F(t), \mathcal{A}_{t}, t \hat{\mathrm{I}} I\right\}$ is a separable square integrable set-valued martingale and for any fixed $\omega \in \Omega, \quad F(\omega, t)$ is lower semicontinuous with $F(0)=0$ a.e., $g$ is a predictable bounded stochastic process. For any $\omega \in \Omega$; $t \hat{\mathrm{I}} I$, define

$$
\begin{aligned}
& (A) \grave{\mathrm{O}}_{0}^{t} g(s, w) d F(s, w) \\
& =\left\{\hat{\mathrm{O}}_{0}^{t} g(s, w) d f(s, w): f ? C M S(F)\right\},
\end{aligned}
$$

then $(A) \mathbf{\mathrm { O }}_{0}^{t} g(s, w) d F(s, w)$ is said to be the Aumann type stochastic integral of $g$ with respect to the set-valued square integrable martingale $F$.

By Therom 4.4 and Therom 4.7 in [6], we have the following Aumann integral measurable theorem:

Theorem 1 Assume that $F=\left\{F(t), \mathcal{A}_{t}, t \hat{\mathrm{I}} I\right\}$ is a separable square integrable set-valued martingale and for any fixed $\omega \in \Omega, F(w, t)$ is lower semicontinuous with $F(0)=0$ a.e., $g$ is a predictable bounded stochastic process. For any $\omega \in \Omega, t \hat{\mathrm{I}} I$, we have that

$$
\begin{aligned}
& c l\left\{(A) \grave{\mathrm{O}}_{0}^{t} g(s, w) d F(s, w)\right\} \\
& =\operatorname{cl}\left\{\hat{\mathrm{O}}_{0}^{t} g(s, w) d f(s, w): f ? C M S(F)\right\}
\end{aligned}
$$

is a set-valued random variable.

The above theorem is useful to discuss the theory of Aumann integral, etc.

\section{Set-Valued Stochastic Integral Equation}

We consider the following set-valued stochastic integral equation

$$
F(t)=F(0)+(L) \int_{0}^{t} b(s, F(s)) d s+(M) \int_{0}^{t} \sigma(s, F(s)) d G(s),
$$

Where for any $t \hat{\mathrm{I}} I, F \hat{\mathrm{I}} L^{2}\left(K\left(R^{d}\right)\right)$ with initial condition $F(0)$ being an $L^{2}$-bounded set-valued random variable, $\quad b: I \times K\left(R^{d}\right) \rightarrow K\left(R^{d}\right)$ is measurable, $\quad \sigma: I \times K\left(R^{d}\right) \rightarrow R^{d} \otimes R^{m} \quad$ is bounded predictable measurable, $G(s)$ is a set-valued square integral martingale. $(L) \mathrm{O}_{0}^{t} b(s, F(s)) d s$ is setvalued stochastic Lebesgue integral (see [9]), $(M) \mathrm{O}_{0}^{t} s(s, F(s)) d G(s)$ is stochastic integral with 
respect to set-valued square integral martingale (see Definition 4.5 in [6]).

Theorem 2 Assume that $b(t, F), s(t, F)$ satisfy the following conditions:

(1) Linear bound condition:

$\|b(t, F)\|^{2}+\|s(t, F)\|^{2} ? a^{2}\left(1 \quad\|F\|_{K}^{2}\right) ;$

(2) Lipschitz continuous condition:

$\left\|b\left(t, F_{1}(t)\right)-b\left(t, F_{2}(t)\right)\right\|+\left\|s\left(t, F_{1}(t)\right)-s\left(t, F_{2}(t)\right)\right\|$ Wa $d_{H}\left(F_{1}(t), F_{2}(t)\right)$

where $a>0$ is a constant;

(3) Set-valued integral inequality:

$$
\begin{aligned}
& E d_{H}^{2}\left(\int_{0}^{t} \sigma\left(s, F_{1}(s)\right) d G_{s}, \int_{0}^{t} \sigma\left(s, F_{2}(s)\right) d G_{s}\right) \\
& \leq E \int_{0}^{t} d_{H}^{2}\left(F_{1}(s), F_{2}(s)\right) d s
\end{aligned}
$$

Then for any $F(0) \hat{\mathrm{I}} L^{2}\left(K\left(R^{d}\right)\right)$, there exists a unique solution to equation (1).

Proof: First, it is obviously the existence of $b, s$ which satisfies (1),(2),(3).

If $F(t) \hat{\mathrm{I}} L^{2}\left(K\left(R^{d}\right)\right)$, for " $t$ ? I, then

$E\|b(t, F(t))\|_{K}^{2}+E\|s(t, F(t))\|^{2} ? a^{2}\left(1 \quad E\|F\|_{K}^{2}\right)$.

Without loss of generality, assume that $b \hat{\mathrm{I}} L^{2}\left(K\left(R^{d}\right)\right)$,

$s$ 文 $L^{2}\left(\begin{array}{ll}R^{d} & R^{m}\end{array}\right)$.

Step 1. $F_{0}(t)=F(0)$,

$$
F_{n+1}(t)=F(0)+\int_{0}^{t} b\left(s, F_{n}(s)\right) d s+\int_{0}^{t} \sigma\left(s, F_{n}(s)\right) d G_{s}, n \geq 0
$$

We shall prove that for any $n^{3} 0, F_{n}$ satisfies:

$$
F_{n} \in L^{2}\left(K\left(R^{d}\right)\right) \text {, for } \forall t \in I
$$

(b) $\quad \lim _{s \circledast t} E d_{H}^{2}\left(F_{n}(t), F_{n}(s)\right)=0$.

For $n=0$, it is obviously right. Suppose that $F_{n}$ has properties $(a),(b)$ for any fixed $n$, we shall prove so does $F_{n+1}$.

Let $Y(t):=\int_{0}^{t} b\left(s_{1}, F_{n}\left(s_{1}\right)\right) d s_{1}$ by Theorem 3.6 in [9], (a) holds to $Y(t)$.

$$
\begin{aligned}
& E d_{H}^{2}(Y(t), Y(s)) \\
& =E d_{H}^{2}\left(c l\left(Y(s)+\grave{\mathrm{o}}_{s}^{t} b\left(s_{1}, F_{n}\left(s_{1}\right) d s_{1}\right), Y(s)\right)\right. \\
& £ E d_{H}^{2}\left(\grave{\mathbf{o}}_{s}^{t} b\left(s_{1}, F_{n}\left(s_{1}\right) d s_{1}, 0\right)\right. \\
& =E\left\|\grave{\mathbf{O}}_{s}^{t} b\left(s_{1}, F_{n}\left(s_{1}\right)\right) d s_{1}\right\|_{K}^{2}
\end{aligned}
$$

By the Linear bound condition (1) and norm property of set-valued Lebesgue integral, it satisfies
Let $Z(t):=\int_{0}^{t} \sigma\left(s_{1}, F_{n}\left(s_{1}\right)\right) d G_{s_{1}}$ It is obviously that (a) holds.

$E d_{H}^{2}(Z(t), Z(s))$

$=E d_{H}^{2}\left(\int_{0}^{t} \sigma\left(s_{1}, F_{n}\left(s_{1}\right)\right) d G_{s_{1}}, \int_{0}^{s} \sigma\left(s_{1}, F_{n}\left(s_{1}\right)\right) d G_{s_{1}}\right)$

$=E d_{H}^{2}\left(\int_{0}^{s} \sigma\left(s_{1}, F_{n}\left(s_{1}\right)\right) d s_{1}+\left(\int_{0}^{t} \sigma\left(s_{1}, F_{n}\left(s_{1}\right)\right) d G_{s_{1}}\right.\right.$

$\left.\left.\Theta \int_{0}^{s} \sigma\left(s_{1}, F_{n}\left(s_{1}\right)\right) d G_{s_{1}}\right), \int_{0}^{s} \sigma\left(s_{1}, F_{n}\left(s_{1}\right)\right) d G_{s_{1}}\right)$

$\leq E d_{H}^{2}\left(\int_{0}^{t} \sigma\left(s_{1}, F_{n}\left(s_{1}\right)\right) d G_{s_{1}} \ominus \int_{0}^{s} \sigma\left(s_{1}, F_{n}\left(s_{1}\right)\right) d G_{s_{1}}\right.$

0)

$\leq E d_{H}^{2}\left(\int_{s}^{t} \sigma\left(s_{1}, F_{n}\left(s_{1}\right)\right) d G_{s_{1}}, 0\right)$

By Theorem 1 and Theorem 4.7 in [6], the setvalued integral Oे $_{\mathrm{s}}^{\mathrm{t}} s\left(s_{1}, F_{n}\left(s_{1}\right)\right) d G_{s_{1}}$ is represented by Aumann integral.

Therefore,

$\lim _{s \otimes t} \mathrm{O}_{\mathrm{s}}^{\mathrm{t}} s\left(s_{1}, F_{n}\left(s_{1}\right)\right) d G_{s_{1}}=\{0\}$, a. e..

Thus, we have $\lim _{s \otimes t} E d_{H}^{2}(Z(t), Z(s))=0$.

Step 2. Now we shall prove that $F_{n}$ convergences to $F$.

Let

$F_{0}(t)=F(0)$,

$F_{n+1}(t)=F\left(t_{0}\right)+\int_{0}^{t} b\left(s, F_{n}(s)\right) d s+\int_{0}^{t} \sigma\left(s, F_{n}(s)\right) d G_{s}, n \geq 0$

We have

$d_{H}\left(F_{1}(t), F_{0}(t)\right)$

$=d_{H}\left(F_{0}(t)+\int_{0}^{t} b\left(s, F_{0}(s)\right) d s+\int_{0}^{t} \sigma\left(s, F_{0}(s)\right) d G_{s}, F_{0}(t)\right)$

$\leq \psi_{H}\left(\int_{0}^{t} b\left(s, F_{0}(s)\right) d s, 0\right)+d_{H}\left(\int_{0}^{t} \sigma\left(s, F_{0}(s)\right) d G_{s}, 0\right)$

$=\left\|\int_{0}^{t} b\left(s, F_{0}(s)\right) d s\right\|_{K}+\left\|\int_{0}^{t} \sigma\left(s, F_{0}(s)\right) d G_{s}\right\|_{K}$

For the first part, we have

$$
\begin{aligned}
E \| \int_{0}^{t} b\left(s, F_{0}(s) d s \|_{K}^{2}\right. & \leq E\left(\int_{0}^{t} \| f\left(s, F_{0}(s) \|_{K} d s\right)^{2}\right. \\
& \leq t E\left(\int_{0}^{t}\left\|b\left(s, F_{0}(s)\right)\right\|_{K}^{2} d s\right) \\
& =t \int_{0}^{t} E\left\|b\left(s, F_{0}(s)\right)\right\|_{K}^{2} d s \\
& \leq t a^{2} \int_{0}^{t}\left(1+E\left\|F_{0}(s)\right\|_{K}^{2}\right) d s \\
& \leq A^{2} t,
\end{aligned}
$$

For the second part, by inequality (3), we have 


$$
\begin{aligned}
& \quad E\left\|\int_{0}^{t} \sigma\left(s, F_{0}(s)\right) d G_{s}\right\|_{K}^{2} \\
& \quad=E d_{H}^{2}\left(\int_{0}^{t} \sigma\left(s, F_{0}(s)\right) d G_{s}, 0\right) \\
& \quad \leq E \int_{0}^{t} d_{H}^{2}\left(F_{0}(s), 0\right) d s \\
& \quad=t E\|F(0)\|_{K}^{2} \\
& \text { Let } B^{2}=E\|F(0)\|_{K}^{2}, \\
& E d_{H}^{2}\left(F_{1}(0), F_{0}(t)\right) \\
& \leq 2 E\left\|\int_{0}^{t} b\left(s, F_{0}(s)\right) d s\right\|_{K}^{2}+2 E\left\|\int_{0}^{t} \sigma\left(s, F_{0}(s)\right) d G_{s}\right\|_{K}^{2} \\
& \leq \\
& \leq
\end{aligned}
$$

By the same way, we have

$$
\begin{aligned}
& E d_{H}^{2}\left(F_{n+1}(t), F_{n}(t)\right) \\
& =E\left(d _ { H } \left(F(0)+\int_{0}^{t} b\left(s, F_{n}(s)\right) d s+\int_{0}^{t} \sigma\left(s, F_{n}(s)\right) d G_{s}, F(0)\right.\right. \\
& \quad+\quad \int_{0}^{t} b\left(s, F_{n-1}(s)\right) d s+\int_{0}^{t} \sigma\left(s, F_{n-1}(s)\right) d G_{s} \\
& \leq E d_{H}(F(0), F(0))+E d_{H}\left(\int_{0}^{t} b\left(s, F_{n}(s)\right) d s, \int_{0}^{t} b\left(s, F_{n-1}(s)\right) d s\right) \\
& \quad+E d_{H}\left(\int_{0}^{t} \sigma\left(s, F_{n}(s)\right) d G_{s}, \int_{0}^{t} \sigma\left(s, F_{n-1}(s)\right) d G_{s}\right) \\
& \leq 2 E d_{H}^{2}\left(\int_{0}^{t} b\left(s, F_{n}(s)\right) d s, \int_{0}^{t} b\left(s, F_{n-1}(s)\right) d s\right) \\
& \quad+2 E d_{H}^{2}\left(\int_{0}^{t} \sigma\left(s, F_{n}(s)\right) d G_{s}, \int_{0}^{t} \sigma\left(s, F_{n-1}(s)\right) d G_{s}\right) \\
& \leq 2 t E \int_{0}^{t} d_{H}^{2}\left(b\left(s, F_{n}(s)\right), b\left(s, F_{n-1}(s)\right)\right) d s \\
& \quad+2 E \int_{0}^{t} d_{H}^{2}\left(F_{n}(s), F_{n-1}(s)\right) d s \\
& \leq 2 t a^{2} E \int_{0}^{t} d_{H}^{2}\left(F_{n}(s), F_{n-1}(s)\right) d s \\
& \quad+2 E \int_{0}^{t} d_{H}^{2}\left(F_{n}(s), F_{n-1}(s)\right) d s \\
& =2\left(t a^{2}+1\right) E \int_{0}^{t} d_{H}^{2}\left(F_{n}(s), F_{n-1}(s)\right) d s
\end{aligned}
$$

Iterating the above process, we get

$$
\begin{aligned}
& E d_{H}^{2}\left(F_{n+1}(t), F_{n}(t)\right) \leq 2^{n+1}\left(t a^{2}+1\right)^{2 n}(A+B)^{2} \frac{t^{n+1}}{(n+1) !} \\
& \text { i.e. } \\
& \qquad \Delta_{n}(t)=E \sup _{s \in[0, t]} d_{H}^{2}\left(F_{n+1}(s), F_{n}(s)\right) \\
& \quad \leq 2^{n+1}\left(t a^{2}+1\right)^{2 n}(A+B)^{2} \frac{t^{n+1}}{(n+1) !}
\end{aligned}
$$

Thus,

$$
\Delta_{n}(T) \leq 2^{n+1}\left(T a^{2}+1\right)^{2 n}(A+B)^{2} \frac{T^{n+1}}{(n+1) !} .
$$

Therefore, we have

$$
\sum_{n=1}^{\infty} \Delta_{n}(T)<\infty
$$

This ensures the existence of the strong solution.
Step 3. Let $F, G$ be two solutions to equation (1). The uniqueness of the solution is the same to the proof of the existence.

Remark 3 Furthermore, fuzzy set-valued martingale integral, fuzzy set-valued stochastic Lebesgue integral [13], and fuzzy set-valued stochastic integral would be useful in the area.

\section{Acknowledgment}

The authors would like to thank reviewers for their valuable comments. This work was supported by youqing NCUT-XN132.

\section{References}

[1] M. Kisielewicz, Differential Inclusions and Optimal Control, Kluwer Academic Publishers, 1991.

[2] I. Karatzas, Lectures on the Mathematics of Finance, American Mathematical Society, Providence, RI, 1997.

[3] A. Fryszkowski, Fixed Point Theory for Decomposable Sets, Kluwer Academic Publishers, 2004.

[4] S. Li and L. Guan, "Fuzzy set-valued Gaussian process and Brownian motion," Information Sciences, vol.177, pp. 3251-3259, August 2007.

[5] J. Li and J. Wang, "On a fluid queue with a fuzzy set-valued Gaussian process input, " unpublished.

[6] S. Li, J. Li and X. Li, "Stochastic integral w.r.t. set -valued square integrable martingale," Journal of Mathematical Analysis and Applications vol.370, pp. 659-671, October 2010.

[7] M. Malinowski, “On a new set-valued stochastic integral with respect to semimartingales and its applications," Journal of Mathematical Analysis and Applications vol.408, pp.669-680, Decembe 2013.

[8] J. Li S. Li and Y. Ogura, "Strong solution of Ito type set-valued stochastic differential equation," Acta Mathematica Sinica, English Series, vol.26, pp. 1739-1748, September 2010.

[9] J. Li and S. Li, "Set-valued stochastic Lebesgue integral and representation theorems, International Journal of Computational Intelligence Systems, ” vol.1, pp. 177-187, May 2008.

[10] J. Li and S. Li, "Ito type set-valued stochastic differential equation, " Journal of Uncertain Systems, vol.3, pp.52-63, February 2009.

[11] M. L. Puri and D. A. Ralescu, "Convergence theorem for fuzzy martingales," Journal of Mathematical Analysis and Applications vol.160, pp.107-122, September 1991.

[12] J. Li, S. Li and Y. Xue, "The Space of Fuzzy Set-Valued Square Integrable Martingales, ” IEEE 
International Conference on Fuzzy Systems, pp. 872-876, August 2009.

[13] J. Li and J. Wang, "Fuzzy set-valued stochastic Lebesgue integral," Fuzzy Sets and Systems, vol.200, pp.48-64, August 2012.

[14] S. Li, Y. Ogura and V. Kreinovich, Limit Theorems and Applications of Set-Valued and Fuzzy Set-Valued Random Variables, Kluwer Academic Publishers, 2002. 